\title{
Factors Associated with the Use of Dietary Supplements among African-American Adults
}

\author{
Romel Franklin, Julie Schneider, and Keiko Goto \\ California State University, Chico \\ Department of Nutrition and Food Sciences
}

\begin{abstract}
The purpose of this study was to examine the prevalence and factors associated with the use of dietary supplements among African-American adults. One hundred and ninety-five (195) African-American adults ages 18 and older completed a self-administered survey that consisted of questions regarding use of supplements, perceived knowledge of supplements, perceived dietary adequacy, sources of information for nutrition and supplement knowledge and demographic information. The overall prevalence of vitamin and mineral use was $63 \%$, the most popular being a multivitamin supplement. The prevalence of nonvitamin and non-mineral supplement use was $41 \%$, the most popular being green tea. Perceived knowledge was a significant factor associated with use; the likelihood of using dietary supplements was significantly higher among those who were perceived to be knowledgeable compared to those who were not knowledgeable. After adjusting for age, sex, income and perceived dietary adequacy, perceived knowledge and education were significantly associated with the use of all types of dietary supplements. The primary sources of supplement knowledge of the participants came from healthcare professionals and the media. Nutrition educators should pay particular attention to the current information sources of the dietary supplements used by their clients.
\end{abstract}

(C) 2009 Californian Journal of Health Promotion. All rights reserved.

Keywords: dietary supplements, perceived knowledge, education, African-American adults

\section{Introduction}

Health disparities between African Americans and other racial/ethnic groups continue to grow in the United States (Underwood et al., 2004). African Americans are more likely to receive poor medical care/attention in the treatment of chronic diseases than other racial groups (Chou et al., 2007). Moreover, African Americans are less likely to be prescribed medication and only a small percentage of them routinely take their prescription medication as compared to other racial groups (Chou et al., 2007). Instead, African Americans tend to self-prescribe dietary supplements, herbs and natural products (Kennedy, 2005). The popularity of dietary supplements may be attributed to ease of accessibility that does not require a prescription and lack of accountability to healthcare professionals on the intended use of the supplement (Gardiner et al., 2007).

In the 2002 National Health Interview Survey of Complementary and Alternative Medicine, dietary supplement use was widespread in the US adult population (Kennedy, 2005). The 2002 Health and Diet Survey presented that $73 \%$ of US adults had taken some form of dietary supplement within the previous 12 months (Timbo, Ross, McCarthy, \& Lin, 2006). Studies reporting the prevalence of supplement use within the African-American population show mixed results. A recent national survey reported that more African Americans (48.7\%) used dietary supplements for weight loss than Hispanics (41.6\%) and Whites (31.2\%) (Pillitteri et al., 2008). Similarly, the high prevalence of dietary supplement use among African- 
Americans was observed in a study in the Jackson, Mississippi metropolitan area; fortynine percent and $66 \%$ of African-American men and women, respectively, used dietary supplements in the study (Talegawkar et al., 2007). Other studies reported the frequent use of dietary supplements, with the most popular being a multivitamin (Gardiner et al., 2007; Pagan \& Pauly, 2005). Supplements such as folk herbal therapies (vinegars, teas, etc.) have also been frequently used among rural African Americans with Type 2 diabetes (Jones et al., 2006). However, in a nationally represented study, African Americans had the lowest prevalence of supplement use with herbal mixtures being particularly uncommon (Gardiner et al., 2007; Kelly, Kaufman, Kelley, Rosenberg, \& Mitchell, 2006). These inconsistent findings may be attributed to the lack of distinction between vitamin/mineral and non-vitamin/non-mineral (NVNM) supplement use and unidentified factors influencing use among African Americans (Jones et al., 2006; Pawlak, Connell, Brown, Meyer, \& Yadrick, 2005; Sternberg, Chandran, \& Sikka, 2003; Weinrich, Hudson Priest, Moyad, \& Weinrich, 2004).

While assessing sex, income, education, and age merit investigation (U.S. Department of Health and Human Services, 2007; Yoon, Horne, \& Adams, 2004), it is important to examine other factors that may be associated with supplement use among African Americans, such as sources of information and perceived knowledge. Historically, African Americans have obtained knowledge from traditional family sources for disease prevention and cure (Jones et al., 2006). It is uncertain to what extent African Americans continue to utilize traditional sources of information for the use of dietary supplements. While it is strongly recommended to obtain evidence-based knowledge from reliable sources, most supplement users, including African Americans, appear to self-prescribe herbs and natural products based on information obtained from non-health care professionals (Kennedy, 2005). Thus, it is important to examine perceived knowledge as a factor affecting dietary supplement use as well as sources of information among African Americans (National Center for Health Statistics [NCHS], 2007). The objectives of this study were, therefore, to (a) assess the prevalence of vitamin/mineral and NVNM supplement use separately among a sample of African-American adults, and (b) examine factors associated with dietary supplement use among a sample of African-American adults.

\section{Methods}

\section{Participants and Settings}

A cross-sectional study was conducted between December 2007 and April 2008. The convenience sample was composed of 195 selfidentified African-American adults, 18 years of age or older, residing in the San Francisco Bay area. Inclusion criteria were adults 18 years of age or older who (a) self-identified themselves as African Americans, (b) resided in any part of the San Francisco Bay Area, and (c) were able to speak, understand and read English. The participants were recruited by verbal announcements at church meetings, personal contacts, community senior organizations, clinics, and churches in the San Francisco Bay area. Informed consent was obtained prior to participation in the study. No direct benefit was available for those who chose to participate. This study was approved by the Institutional Review Board at California State University, Chico.

\section{Survey Instruments}

The validated survey instrument from a previous study conducted by Newberry and colleagues (Newberry, Beerman, Duncan, McGuire, \& Hillers, 2001) was used for this study. The format of the questions included forced-choice, five-point Likert scale, and open-ended responses. The survey was reviewed by nutrition professionals including an African-American nutritionist for readability and content validity. The survey was pilot tested with 44 African Americans prior to study recruitment to gauge readability and comprehension. No issues were identified based on the pilot study, and no modifications were made to the survey instrument. 
Subjects completed a self-administered survey that assessed use of supplements, perceived knowledge of supplements, perceived dietary adequacy, sources of information for nutrition and supplement knowledge, use of prescription medication, and prevalence of chronic diseases. Demographic data were also assessed for years of education, income, sex, age, height and weight. Self-reported height and weight were used to compute the participant's body mass index (BMI = weight in $\mathrm{kg} /$ height in meters squared). In addition, the survey used an extensive list of non-vitamin, non-mineral supplements to assess intake in the last 12 months. The survey took approximately 10 to 15 minutes to complete and was directly returned to the first author or was mailed to the Department of Nutrition and Food Sciences at California State University, Chico.

\section{Statistical Analyses}

Descriptive statistics were performed to identify the demographic characteristics of the participants, types and frequency of dietary supplements used, perceived knowledge, perceived dietary adequacy, and sources of information for nutrition and supplement knowledge. Chi-square tests were used to compare proportions and t-tests were used to compare means between supplement users and non-users so as to determine factors associated with the use of supplements. Multiple logistic regression models were developed, and adjusted odds ratios (ORs) were used to evaluate factors associated with the use of vitamins or minerals (vitamin/mineral model) or the use of NVNM supplements (NVNM model). We selected characteristics for testing logistic regression models based on the results of previous national studies as well as nearly significant detections in our statistical analyses. Therefore, age, sex, income, education, perceived knowledge, and perceived dietary adequacy were included in the multiple logistic regression models. Categorical variables were dichotomized for the multiple logistic regression analyses. Data were analyzed using SPSS (version 15.0 for Windows, SPSS Inc., Chicago, IL, 2006). A p-value of less than or equal to 0.05 was set as the level of significance.

\section{Results}

\section{Characteristics of Participants}

The demographic variables of the study participants are listed in Table 1. Of the 195 participants completing the study, approximately $70 \%$ were women. Most participants (67\%, $\mathrm{n}=131$ ) had some college experience or earned a college degree. Many of our study participants earned between $\$ 35,000$ - $\$ 64,999(31 \%, n=61)$. Mean BMI for women was $27.9( \pm 6.82)$ with a range of 16.7 to 55.3, and mean BMI for men was $27.3( \pm 5.97)$ with a range of 17.7 to 45.6 . In addition, $73 \%$ of the study participants described their perceived health status as good or excellent while $20 \%$ perceived it to be fair (data not shown). Only $4.6 \%$ of the study participants perceived their health status was poor (data not shown).

Table1. Demographic Characteristics of African-American Study Participants

\begin{tabular}{lcc}
\hline \hline Characteristic & $\boldsymbol{n}$ & Percentage \\
\hline \hline Sex & 58 & 29.7 \\
Male & 133 & 68.2 \\
Female & 4 & 2.1 \\
Missing & & \\
\hline Age in years & 38 & 19.5 \\
18-24 & 32 & 16.4 \\
$25-34$ & 37 & 19.0 \\
$35-44$ & 27 & 13.8 \\
$45-54$ & 33 & 16.9 \\
55-64 & 22 & 11.3 \\
65 and older & 6 & 3.1 \\
Missing & & \\
\hline Education & 1 & 0.5 \\
Less than high school & 5 & 2.6 \\
Some high school & 54 & 27.7 \\
High school degree or GED & 72 & 36.9 \\
Some college & 59 & 30.3 \\
College graduate & 4 & 2.1 \\
Missing & & \\
Annual Income & 41 & 21.0 \\
Less than $\$ 15,000$ & 50 & 25.6 \\
\$15,000 to $\$ 34,999$ & 61 & 31.3 \\
\$35,000 to \$64,000 & 26 & 13.3 \\
Greater than \$64,000 & 17 & 8.7 \\
Missing & & \\
\hline \hline
\end{tabular}


The prevalence of vitamin or mineral supplement use, as well as non-vitamin nonmineral (NVNM) supplement use, is presented in Table 2. The use of vitamins or minerals in the prior 12 months was $63 \% \quad(n=119)$. Significantly more women used vitamin/mineral supplements than men $(\mathrm{p}=0.002)$. The majority of participants $(59 \%, \mathrm{n}=111)$ reported the use of vitamin/mineral supplements within the past year. The most common vitamin/mineral supplements used were multivitamin preparations such as One a Day ${ }^{\circledR}$, GNC ${ }^{\circledR}$ brands, and Centrum ${ }^{\circledR}$. Among the vitamin/mineral supplement users, 39\% $(n=74)$ reported taking multivitamins daily, $6.8 \%$ $(n=13)$ reported weekly usage, $0.5 \% \quad(n=1)$ reported monthly usage, and $3.7 \% \quad(n=7)$ reported less frequent usage (e.g., less than monthly).

Table 2. Percentage and P-Values (Chi-Square or Linear-by-Linear Association) of Participant Characteristics between Vitamin/Mineral Supplement Users and NVNM $^{1}$ Supplement Users

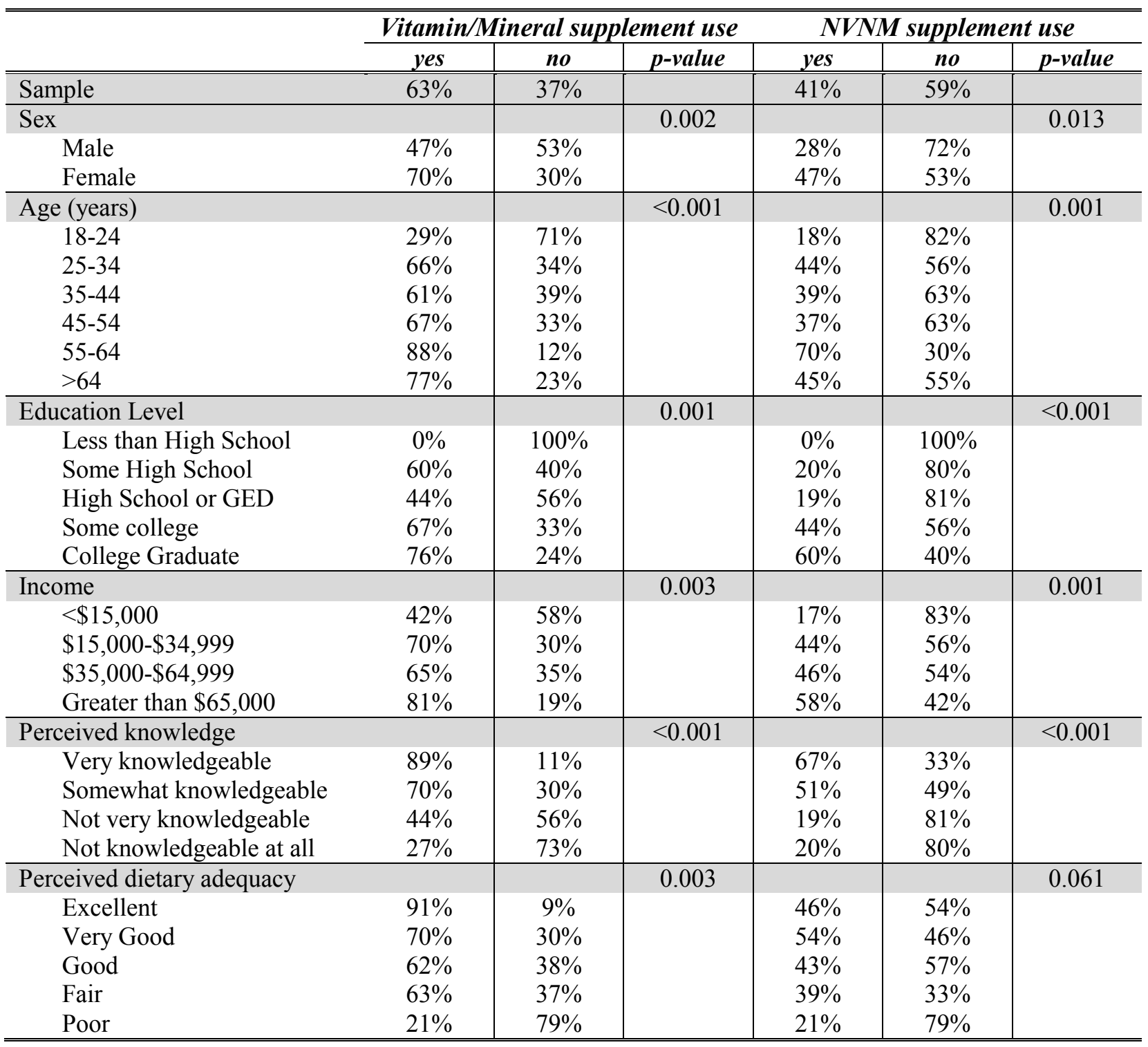


Among the 195 participants, less than half $(41 \%$, $\mathrm{n}=77$ ) reported use of NVNM supplements within the past 12 months. The most common NVNM dietary supplements were green tea, fish oil, fiber, flaxseed, cranberry and chamomile.

\section{Factors Associated with Supplemental Use}

Table 2 presents percentage and p-values of participant characteristics between vitamin/mineral supplement users and NVNM supplement users. Supplement use was higher among those who were older, with a higher level of education, and with a higher income level. In addition to age, sex, income and education, perceived knowledge of dietary supplements and perceived dietary adequacy were significantly positively associated with the use of vitamin/mineral supplements $(\mathrm{p}<0.001$ and $\mathrm{p}=0.003$, respectively). As for factors associated with NVNM use, women and older age groups were significantly more likely to use NVNM supplements than men and younger groups respectively. The income and educational levels of the study participants were also significantly associated with the use of NVNM supplements. Similar to vitamin/mineral use, perceived knowledge was significantly associated with the use of NVNM supplements $(\mathrm{p}<0.001)$ while perceived dietary adequacy was not a significant factor $(0.061)$.

Table 3. Adjusted Odds Ratios (95\% Confidence Intervals) for Factors Associated with Use of Vitamin/Mineral Supplements and Non-Vitamin/Non-Mineral Supplements in African-American Adults

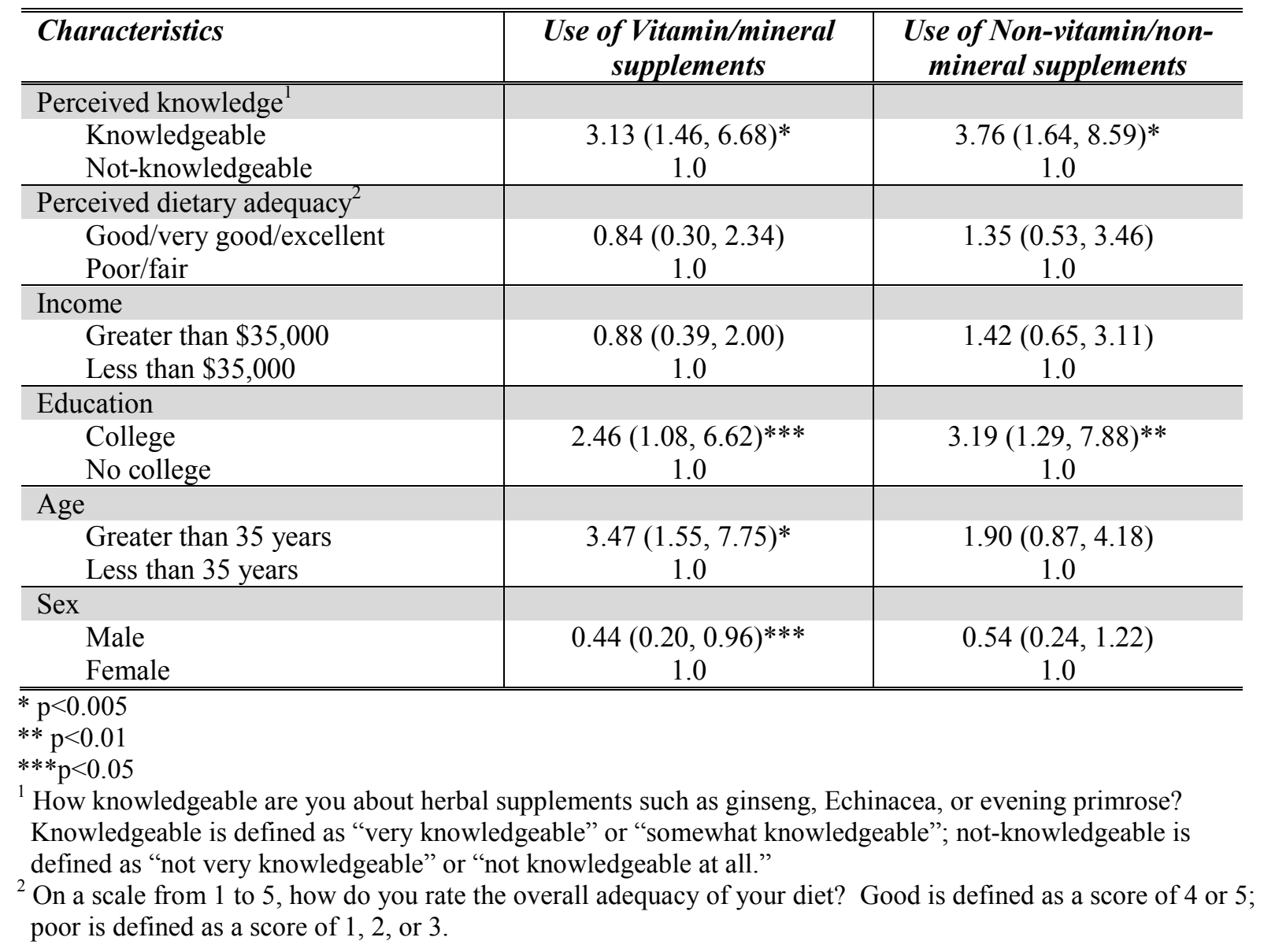




\section{Multi-variable Analysis of Factors Associated with Supplement Use}

Adjusted odds ratios for factors associated with supplement use are presented in Table 3. The odds of using vitamin/mineral supplements were significantly greater among participants with higher perceived knowledge $(\mathrm{OR}=3.13$, $\mathrm{P}=0.003$ ) after adjusting for age, sex, income, education and perceived dietary adequacy. Other factors independently associated with the use of vitamin/mineral supplements included age $(\mathrm{OR}=3.47, \mathrm{p}=0.002)$, sex $(\mathrm{OR}=0.44, \mathrm{P}=0.040)$ and education $(\mathrm{OR}=2.46, \mathrm{P}=0.033)$.

Factors significantly associated with the use of NVNM supplements were perceived knowledge $(\mathrm{OR}=3.76, \mathrm{P}=0.002)$ and higher levels of education $(\mathrm{OR}=3.19, \mathrm{P}=0.012)$ after controlling for age, sex, income and perceived dietary adequacy. On the other hand, age and sex were no longer significant factors associated with the use of NVNM supplements after controlling for the other variables.

\section{Information Sources}

Participants reported that the majority of their knowledge about dietary supplements came from health care professionals $(48 \%)$, such as physicians, pharmacists, and dietitians. The second greatest source of knowledge was the media (45\%), followed by food and supplement labels (38\%), nutrition specialty stores $(29 \%)$, the internet (24\%), and athletic coaches $(10 \%)$ (data not shown). Other sources of knowledge reported were family, friends, school, books, anecdotal accounts, and self-usage. Approximately one-fifth of the participants $(20 \%)$ reported that they did not know much about dietary supplements.

Participants reported that the most useful health and nutrition information was obtained from a physician $(48 \%)$, the media $(46 \%)$, friends and relatives $(41 \%)$, nutrition professionals $(35 \%)$, the internet $(29 \%)$, a supplement store $(27 \%)$ and a coach (8.7\%) (data not shown).

\section{Discussion}

Little is known about the prevalence of and factors associated with the use of various types of dietary supplements, especially NVNM, among African Americans, one of the high-risk groups for developing chronic diseases in the United States. Previous studies combined vitamin/mineral and NVNM supplements or focused on only one supplement type to assess prevalence (Gardiner et al., 2007; Hirayama, Lee, Binns, Watanabe, \& Ogawa, 2008; Newberry et al., 2001; Pillitteri et al., 2008; Stasio, Curry, Sutton-Skinner, \& Glassman, 2008), particularly among African Americans (Jones et al., 2006; Pawlak et al., 2005; Sternberg et al., 2003; Weinrich et al., 2004), possibly contributing to the inconsistency of study results. To our knowledge, this is the first study that investigated the prevalence of and factors associated with the use of vitamin/mineral and NVNM supplements separately among African-American adults, particularly focusing on perceived knowledge as a factor affecting dietary supplement use.

Vitamin/mineral supplement use was quite high among both men (47\%) and women $(70 \%)$ in our study population. This finding was consistent with the study by Weinrich and colleagues (2004), who exclusively studied African-American men ages 40-70 years. The authors found over half $(51 \%)$ took at least one type of dietary supplement, the most popular being a multivitamin supplement (46\%). However, the subjects in that study were attending a prostate cancer educational and screening program, and the high percentage of men using supplements may be attributed to their interest in health (Weinrich et al., 2004). Thus, our study findings suggest that multivitamins may be a common type of vitamin/mineral supplement among apparently healthy African-American adults as well.

NVNM supplement use, such as herbal products, was lower $(41 \%)$ than vitamin/mineral supplement use $(63 \%)$ in our study. This is in agreement with other studies that suggest the overall prevalence of NVNM supplements among African Americans tends to be low, especially with regards to herbal dietary supplements (Sternberg et al., 2003). In a nationally represented study, it was found that herbal mixtures were particularly uncommon 
among African Americans (Kelly et al., 2006). Our study participants reported green tea as the most popularly used supplement, followed by fish oil, fiber, flaxseed, and cranberry supplements. In a study conducted from 1998 to 2004, lutein was the most common NVNM supplement (2.4\%), followed by Panax ginseng $(1.7 \%)$ and garlic (1.5\%) (Kelly et al., 2006). In another study, among 57 African-American seniors aged 65 and over, the most common supplements taken were glucosamine $(26.3 \%)$, garlic $(26.3 \%)$, aloe and ginkgo biloba (both $15.8 \%$ ) (Sternberg et al., 2003). Further research is needed to understand the relationships between demographic or health characteristics and types of NVNM supplements.

Factors associated with dietary supplement use were examined in our study. Previous studies have shown that the majority of supplement users tend to be older or college educated females with relatively higher incomes (Newberry et al., 2001; U.S. Department of Health and Human Services, 2007). Only one study reported that men tended to use more herb/botanical-based supplements than women (Gardiner et al., 2007). On the other hand, other important factors such as perceived knowledge have not been well examined. We found that perceived knowledge, education, age, and sex were independently associated with the use of vitamins/minerals, while perceived knowledge and education were associated with the use of NVNM. Perceived knowledge and education were associated with overall supplement use among this African-American sample.

Interestingly, the majority of the study participants received their dietary supplement knowledge and nutrition information from qualified health professionals $(48 \%)$ and the media (45\%). The sources from professionals are usually scientifically based while anecdotal sources such as family, friends, and the media may not be. Several other studies that examined influencing factors of dietary supplement among African Americans identified these anecdotal sources such as friends, neighbors, and relatives as primary influencing factors (Sternberg et al., 2003; Yoon et al., 2004). Further studies are needed to examine the accuracy of perceived knowledge among African-American adults, as well as sources of information for particular supplements such as herbs.

We also found that perceived dietary adequacy was positively related to the use of vitamin/mineral supplements, although it became insignificant after controlling for other variables. Typically good and balanced diets provide what mainstream multivitamin supplements contain with the exception of multivitamins that contain herbs and spices. It is possible that many of the supplement users' intent is to exceed vitamin/mineral intake recommendations in order to provide even "better" nutrition for their bodies. Another study reported that participants with healthier lifestyles were more likely to use dietary supplements (Foote et al., 2003). On the other hand, some of the vitamin/mineral supplement users may think diet plays a less important role in meeting their recommended Dietary Reference Intakes for those vitamins/minerals. Further research is needed to examine the relationship between dietary adequacy and supplement use.

This study has several limitations. Dietary supplement use was self-reported, allowing under- or over-reported use. For example, many of today's popular multivitamins and energy drinks contain herbs (e.g., ginseng, ginkgo etc.), and our study participants may not have been aware of it. Under-reporting could have occurred due to various definitions of supplements. Some participants may not have considered teas as dietary supplements. Second, although perceived knowledge and dietary adequacy played important roles in supplement use in our study, the scientific accuracy of the perceived knowledge or actual diet adequacy were not measured. Thus, it is uncertain whether actual scientific knowledge or diet adequacy might have been associated with the use of supplements. Third, about $31 \%$ of study participants, who were self-identified African Americans, had earned a bachelor's degree while only $17.3 \%$ of non-Hispanic Blacks attained a bachelor degree, according to the U.S. Census Bureau, in 2000 (Weinrich et al., 2004). Our study findings suggest that higher education is associated with more supplement use and, 
therefore, the use of dietary supplements in our study population might have been higher than the general African-American population. The relationship between education and supplement use needs to be further explored.

\section{Conclusion}

The use of dietary supplements has continually increased over the last three decades in the United States (Kelly et al., 2006). Our findings will significantly contribute to the nutrition community, particularly nutrition practitioners who provide education regarding dietary supplements to African Americans; a high-risk population in the United States. In our study, African Americans tended to use more vitamin/mineral than NVNM supplements and the use of both types of supplements was independently associated with perceived knowledge and education after controlling for age, sex and income. Furthermore, among our study participants the majority of perceived knowledge came from health care professionals and the media, which are different from the traditional knowledge sources passed down from generation to generation in the AfricanAmerican community. This may possibly reflect the influence of higher educational levels, as found in our study. For their evidence-based nutrition practices, nutrition educators should pay particular attention to the current information sources of the dietary supplements used by their African-American clients.

The results of this study provide an important perspective of supplement use among the underserved African Americans. Future studies are needed to further examine relationships between types of dietary supplements, perceived knowledge among African Americans and sources of information regarding dietary supplements.

\section{Acknowledgements}

The study was supported by the Department of Nutrition and Food Sciences at California State University, Chico. No competing financial interests exist.

\section{References}

Chou, A. F., Brown, A. F., Jensen, R. E., Shih, S., Pawlson, G., \& Scholle, S. H. (2007). Gender and racial disparities in the management of diabetes mellitus among Medicare patients. Women's Health Issues, 17, 150-161.

Foote, J. A., Murphy, S. P., Wilkens, L. R., Hankin, J. H., Henderson, B. E., \& Kolonel, L. N. (2003). Factors associated with dietary supplement use among healthy adults of five ethnicities: the Multiethnic Cohort Study. American Journal of Epidemiology, 157, 888-897.

Gardiner, P., Graham, R., Legedza, A. T., Ahn, A. C., Eisenberg, D. M., \& Phillips, R. S. (2007). Factors associated with herbal therapy use by adults in the United States. Alternative Therapy in Health and Medicine, 13, 22-29.

Hirayama, F., Lee, A. H., Binns, C. W., Watanabe, F., \& Ogawa, T. (2008). Dietary supplementation by older adults in Japan. Asia Pacific Journal of Clinical Nutrition, 17, 280-284.

Jones, R. A., Utz, S., Wenzel, J., Steeves, R., Hinton, I., Andrews, D., et al. (2006). Use of complementary and alternative therapies by rural African Americans with type 2 diabetes. Alternative Therapy in Health and Medicine, 12, 34-38.

Kelly, J. P., Kaufman, D. W., Kelley, K., Rosenberg, L., \& Mitchell, A. A. (2006). Use of herbal/natural supplements according to racial/ethnic group. Journal of Alternative and Complementary Medicine, 12, 555-561.

Kennedy, J. (2005). Herb and supplement use in the US adult population. Clinical Therapy, 27, 18471858.

National Center for Health Statistics (NCHS). (2007). National Center for Health Statistics Hyattsville, MDo. Document Number)

Newberry, H., Beerman, K., Duncan, S., McGuire, M., \& Hillers, V. (2001). Use of nonvitamin, nonmineral dietary supplements among college students. Journal of American College Health, 50, 123-129. 
Pagan, J. A., \& Pauly, M. V. (2005). Access to conventional medical care and the use of complementary and alternative medicine. Health Affairs, 24, 255-262.

Pawlak, R., Connell, C., Brown, D., Meyer, M. K., \& Yadrick, K. (2005). Predictors of multivitamin supplement use among African-American female students: a prospective study utilizing the theory of planned behavior. Ethnicity and Disease, 15, 540-547.

Pillitteri, J. L., Shiffman, S., Rohay, J. M., Harkins, A. M., Burton, S. L., \& Wadden, T. A. (2008). Use of dietary supplements for weight loss in the United States: results of a national survey. Obesity, 16, 790-796.

Stasio, M. J., Curry, K., Sutton-Skinner, K. M., \& Glassman, D. M. (2008). Over-the-counter medication and herbal or dietary supplement use in college: dose frequency and relationship to self-reported distress. Journal of American College Health, 56, 535-547.

Sternberg, S. A., Chandran, A., \& Sikka, M. (2003). Alternative therapy use by elderly African Americans attending a community clinic. Journal of the American Geriatrics Society, 51, 17681772.

Talegawkar, S. A., Johnson, E. J., Carithers, T., Taylor, H. A., Jr., Bogle, M. L., \& Tucker, K. L. (2007). Total alpha-tocopherol intakes are associated with serum alpha-tocopherol concentrations in African American adults. Journal of Nutrition, 137, 2297-2303.

Timbo, B. B., Ross, M. P., McCarthy, P. V., \& Lin, C. T. (2006). Dietary supplements in a national survey: Prevalence of use and reports of adverse events. Journal of the American Dietetic Association, 106, 1966-1974.

U.S. Department of Health and Human Services. (2007). National Health and Nutrition Survey o. Document Number)

Underwood, S. M., Buseh, A. G., Canales, M. K., Powe, B., Dockery, B., Kather, T., et al. (2004). Nursing contributions to the elimination of health disparities among African-Americans: review and critique of a decade of research. Journal of the National Black Nurses Association, 15, 48-62.

Weinrich, S. P., Hudson Priest, J., Moyad, M. A., \& Weinrich, M. C. (2004). Intake of selected nutritional supplements by African-American men. Urology, 64, 1094-1097.

Yoon, S. L., Horne, C. H., \& Adams, C. (2004). Herbal product use by African American older women. Clinical Nurses Research, 13, 271-288.

Author Information

Romel Franklin, M.S.

California State University, Chico

Department of Nutrition and Food Sciences

Julie Schneider, Ph.D.

Assistant Professor

California State University, Chico

Department of Nutrition and Food Sciences

*Keiko Goto, Ph.D.

Assistant Professor

Department of Nutrition and Food Sciences

California State University, Chico

100 West 1st St.

Chico, CA 95929-0002

Phone: 530-898-6767

Fax: 530-898-5586

E-mail: kgoto@csu.chico.edu

* corresponding author 